\title{
Experiencias
}

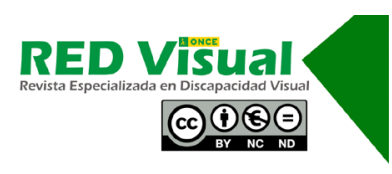

Recepción: 05-03-2021 Aceptación: 22-03-2021

\section{Experiencia de trabajo social en la ONCE en Madrid ante una situación de crisis sanitaria: intervención y aprendizajes}

\author{
Social work experience at ONCE, Madrid, \\ in the context of a health crisis: \\ action taken and lessons learnt
}

\author{
V. Felipe López, L. Fernández Sanz, M. C. Jiménez Cruz, \\ M. J. Martínez Vitoria, G. Mendoza Mendoza, J. Olalla Suárez, \\ M. T. Puerto Simón
}

\section{Resumen}

El objetivo de este trabajo es dar a conocer la experiencia del equipo de trabajadoras sociales de la ONCE de la Delegación Territorial de Madrid durante el periodo de confinamiento derivado de la pandemia del SARS-CoV-2, así como después del mismo, además de una reflexión sobre el trabajo realizado y los aprendizajes obtenidos. Ha sido una experiencia donde se han atendido las necesidades sociales con un nuevo enfoque ante la incertidumbre generada por la situación sanitaria. Se inicia la experiencia con información relacionada con los inicios del confinamiento y la incertidumbre, así como los procedimientos y las tareas en una situación diferente, en la que los profesionales realizaban su intervención con grandes dosis de motivación e imaginación para, después, abordar la situación de «nueva normalidad» con una atención preferentemente telefónica, escasos y excepcionales contactos presenciales, con el uso de la tecnología como apoyo a todos los procesos, los cuales, como consecuencia de la sobrecarga de la administración, se fueron alargando. Más tarde, se enumeran los aprendizajes obtenidos, las propuestas del cambio en la intervención con las personas y con el propio equipo de trabajo social, reforzando la idea del cuidado profesional y el empoderamiento de las personas usuarias. 


\title{
Palabras clave
}

Trabajo social. Bienestar. Necesidades básicas. Estado anímico. Adaptación. Vulnerabilidad. Acompañamiento.

\begin{abstract}
This article describes the experience of the social counsellors working out of the ONCE'S community centre at Madrid during and after the SARS-CoV-2 lockdown, analysing the work performed and the lessons learnt. The experience consisted in the adoption of a new approach to meet social needs to confront the uncertainty generated by the health crisis. It began with the collection of information on the uncertainty surrounding early-stage lockdown and the roll-out of new procedures and tasks in an unprecedented situation that called upon professionals to deploy huge doses of motivation and imagination. The 'new normality' was confronted primarily via telephone support and scant and only exceptional in-person contact. Professionals resorted to technology to expedite all the processes that, due to the heavy administrative burden, were experiencing delays. That description is followed by a discussion of the lessons learnt, proposals for change in the support afforded affiliates and in the social counsel team itself to reinforce professionalised care and user empowerment.
\end{abstract}

\section{Key words}

Social work. Welfare. Basic needs. State of mind. Adaptation. Vulnerability. Companionship.

\section{Trabajo social de la ONCE: servicio de apoyo al bienestar social}

Tal y como menciona el Código deontológico del trabajo social (Consejo General del Trabajo Social, 2015) en su artículo 17:

Los profesionales del trabajo social se comprometen en la intervención social a buscar y garantizar a toda persona, grupo o comunidad la igualdad de oportunidades, el acceso a recursos y el apoyo para cubrir sus necesidades; especialmente de aquellos que se encuentran en situación de mayor vulnerabilidad o en alguna situación específica de desventaja social. 
En nuestro ámbito de actuación -la ONCE-, la atención a las personas afiliadas viene definida por un modelo específico de servicios sociales, el cual establece los requisitos básicos de calidad que debe reunir su actividad, así como la descripción del proceso de prestación de servicios.

El trabajador social en esta institución desarrollaría su ámbito de actuación dentro del servicio de apoyo al bienestar social. Pero antes, ¿qué podemos entender por bienestar social?

Muchos son los autores que han definido este concepto y muchas son las similitudes con otros términos, como acción social, servicios sociales, trabajo social, intervención social, aunque son cuestiones totalmente diferentes, ya que unos están relacionados con los medios y otros con la acción propiamente dicha.

A modo de ejemplo, Pérez (2019, p. 20) cita las palabras de Ander-Egg, que define este concepto como «toda actividad consciente, organizada y dirigida de manera individual o colectiva, que de modo expreso tiene por finalidad actuar sobre el medio social, para mantener una situación, mejorarla o transformarla».

Se podría decir que el bienestar social es un valor social que tiene como finalidad última la cobertura de las necesidades sociales de los individuos mediante los medios que la sociedad pone a su alcance.

Es el objeto de una acción elaborada y consecuencia de una reflexión, planificación y evaluación, con el objetivo de conseguir los mejores niveles de calidad de vida para las personas que presentan necesidades básicas.

El bienestar social, en muchas ocasiones, ha sido relacionado con los conceptos de calidad de vida o situación económica, pero no necesariamente son lo mismo. Además, es preciso tener en cuenta la visión subjetiva de lo que cada uno percibe sobre lo que es el bienestar.

Volviendo al modelo de servicios sociales de la ONCE, la función del trabajo social abarca dos fases transcendentes. La primera de ellas es la fase de solicitud de afiliación a la ONCE, informando sobre este proceso, la finalidad de la institución y, de forma general, sobre los recursos y servicios de la entidad y de otras instituciones. 
La segunda fase, de planificación, es aquella centrada en la persona, en la que se realiza la primera atención a la misma, con la valoración de su situación personal, familiar y relacional, la elaboración del diagnóstico social, finalizando con la propuesta del plan individualizado de atención.

Dentro de esta fase, podemos definir el apoyo al bienestar social como el servicio que garantiza a las personas afiliadas unas condiciones elementales de calidad de vida y de acceso a los diferentes recursos esenciales y/o de la comunidad con el objetivo de fomentar su autonomía e integración social (Zamanillo, 1989).

\section{Primera fase: frente al desconocimiento}

El 13 de marzo de 2020 y, tras la publicación del Real Decreto 463/2020, se declaró en nuestro país el estado de alarma, con el objetivo principal de prevenir el contagio por un nuevo virus que fue descubierto a finales del 2019 en China y que se había ido propagando por diferentes países hasta llegar a España: el SARS-CoV-2.

La rápida propagación de este por nuestro país supuso la saturación de los servicios sanitarios, que se vieron sobrepasados por la necesidad de atender a pacientes con covid y carecer de medios suficientes para ello.

Inicialmente, este estado de alarma estaba previsto que durase 15 días, aunque luego no fue así. Esto obligó a permanecer confinados a los ciudadanos en sus domicilios, exceptuando la realización de compras de primera necesidad o acudir al puesto de trabajo, suspendiendo el resto de actividades.

El 28 de marzo se endurece la cuarentena, obligando a todos los trabajadores de servicios no esenciales a permanecer en sus casas entre el 30 de marzo y el 9 de abril.

Finalmente, la situación de excepción se va prorrogando quincenalmente hasta el 10 de mayo; aunque seguíamos bajo el paraguas del estado de alarma, las medidas adoptadas por el Estado y las diferentes comunidades autónomas se van relajando y se empiezan a permitir las salidas controladas y organizadas del domicilio. Estas varían en función de los datos de contagio de provincias o municipios. 
El 21 de junio, al acabar el estado de alarma, todos los territorios pasan a la denominada nueva normalidad a través del proceso conocido como desescalada, que consistía en una serie de fases en las que se iban relajando las medidas en función de la incidencia de contagios en los diferentes territorios.

\subsection{Metodología de intervención en fase de confinamiento}

En este contexto, nuestra actividad y funciones fueron evolucionando según las circunstancias de cada momento.

El 16 de marzo de 2020, con gran incertidumbre, acudimos a nuestros centros de trabajo para recoger las herramientas e indicaciones necesarias para desarrollar nuestra labor desde nuestros domicilios durante los 15 días posteriores.

En un principio, se realizaban llamadas de seguimiento a aquellos afiliados mayores que vivían solos para hacer una valoración de necesidades, así como atender las demandas realizadas por los afiliados que acudían a la ONCE en busca de apoyos.

Durante este periodo, las atenciones se enfocaron principalmente en facilitar información y apoyar en el acceso a los afiliados a recursos básicos de alimentación, farmacéuticos o incluso sanitarios.

Con la primera ampliación del confinamiento y el endurecimiento de las medidas, comienzan a surgir necesidades diferentes:

- Problemas económicos derivados de las dificultades en el cobro de las prestaciones por un ERTE.

- Consecuencias socioeconómicas negativas derivadas de la imposibilidad de acudir al puesto de trabajo para aquellas personas que trabajaban en empleo sumergido.

- La soledad y la incertidumbre ante el futuro, e incluso ante el presente.

Las necesidades surgidas en este periodo se alargan durante todo el confinamiento, observándose un crecimiento paulatino en el número de personas afiliadas que solicitaban ayuda para el acceso a recursos económicos, y que precisaban acompañamiento y contención emocional. 
A partir del 10 de mayo, con la denominada desescalada, comienzan a surgir en nuestro colectivo miedos diferentes vinculados a las limitaciones que se presentan a la hora de tomar las medidas de prevención y distanciamiento para garantizar su propia seguridad. Miedos alentados por las continuas noticias en los medios de comunicación sobre fallecimientos, formas de contagios e incumplimientos de las normas, que sobreinformaban o facilitaban información variable en función de la evolución de la pandemia.

Durante todo este periodo, las trabajadoras sociales nos vimos empujadas a una adaptación constante a los sucesivos cambios en las restricciones y normas, haciendo frente a las limitaciones en el acceso a recursos y los cambios normativos de las diferentes administraciones.

Desarrollamos la capacidad de adaptación a una situación totalmente desconocida, tanto en lo personal como en lo profesional, intentando llegar a todas nuestras personas afiliadas, en especial a las más vulnerables. Hemos reformulado nuestra intervención profesional, dejando a un lado las técnicas y estrategias habituales (entrevistas presenciales, visitas a domicilio, intervenciones grupales) y adaptándonos a la implantación forzosa del teletrabajo, que supuso utilizar la entrevista telefónica y el correo electrónico como las únicas formas de intervención e interacción con nuestros usuarios, observando que en ese momento daban resultados satisfactorios a la vez que manifestaban la brecha tecnológica según los sectores sociales, culturales y de la edad.

En este sentido, también nos encontramos con numerosas dificultades para acceder a la Administración pública que se encontraba desbordada y sin los medios telemáticos necesarios. Tuvimos que facilitar y favorecer el acceso de nuestros afiliados y sus familias a las alternativas que posibilitaban la consecución de esta documentación, necesaria tanto para la tramitación de los expedientes de prestaciones públicas como del sistema de prestaciones de la ONCE.

Dicho estado de alarma nos obligó a replantear objetivos operativos, llevando a cabo intervenciones profesionales ajustadas a la nueva situación y adaptando las actuaciones a las necesidades que fueron surgiendo en los distintos momentos, siempre teniendo en cuenta el estado emocional de aquellas personas que íbamos atendiendo.

Quizás, como labor más reseñable se podría destacar la de acompañar a nuestros afiliados en los miedos derivados de esta situación, miedos que, a nivel personal, nosotras también sentíamos. 
Durante el periodo de confinamiento más estricto nos volcamos en ofrecer contención emocional y acompañamiento a aquellos afiliados que estaban o se sentían solos, que, en su mayoría, eran personas mayores en sus domicilios o en residencias, donde esta situación se vivió con un halo de incertidumbre y aislamiento añadido.

A raíz del inicio de la desescalada, este apoyo emocional se centró en trasladar seguridad y potenciar los recursos personales de nuestros afiliados para afrontar los nuevos miedos surgidos a causa de la autorización de las salidas de los domicilios. Miedos que surgen en nuestro colectivo, añadidos a los que todos experimentábamos, debido a las limitaciones vinculadas a la discapacidad visual que dificultaba la adaptación a la nueva forma de interacción social.

Como dato significativo, destacar la limitación de las personas con ceguera o discapacidad visual grave a la hora de tocar, explorar, recibir información y referencias a través de sus manos y el contacto físico, funcionamiento prohibido y altamente peligroso.

Todos los conocimientos obtenidos en esta etapa nos han sido útiles y nos han facilitado la adaptación a las nuevas formas de trabajo en las fases posteriores que impone la realidad actual.

Estamos plenamente convencidas de que serán de utilidad en los tiempos que están por venir.

\section{Segunda fase: después del confinamiento, la «nueva normalidad»}

Tras la reincorporación en la fase de «nueva normalidad», se detectaron diferentes aspectos en cuanto a la atención y la modificación de la misma, con sus normas específicas.

Se realizaban atenciones presenciales de forma excepcional, continuando con la atención telefónica de forma prioritaria, pese a que, a veces, tuvimos que combinar ambos modelos.

Todo esto ha conllevado la adaptación de la técnica de la entrevista, a través de la cual obtenemos y proporcionamos información, así como establecemos una relación.

Felipe, V., Fernández, L., Jiménez, M.C., Martínez, M. J., Mendoza, G., Olalla, J., y Puerto, M.T. (2021). Experiencia de trabajo social en la ONCE en Madrid ante una situación de crisis sanitaria: intervención y aprendizajes. RED Visual: Revista Especializada en Discapacidad Visual, 77, 158-171. https://doi.org/10.53094/DJZE9509. 
Nos ha llevado a adecuar nuestras habilidades comunicativas, puesto que, en una entrevista presencial, se puede establecer comunicación no verbal con la mirada, postura corporal o con los gestos que realizamos y que ayudan a trasmitir mensajes de confianza y empatía, proporcionando una información valiosa para la elaboración del diagnóstico social y la planificación de la intervención.

Dentro de las escasas intervenciones presenciales, se ha tenido que limitar el contacto físico, teniendo en cuenta que dicho contacto, en el colectivo de personas con deficiencia visual grave, era un elemento más del apoyo emocional esencial enmarcado en nuestra intervención.

Otro aspecto a tener en cuenta con la implantación de la entrevista telefónica, es la influencia del entorno donde se encuentra la persona atendida, si tiene un espacio de confort, de confidencialidad, en el que pueda expresar su demanda o explorar sus preocupaciones sin interrupciones.

Se han tenido que replantear prioridades, ya que continuamos en una situación de crisis social, económica y relacional, donde los modelos de intervención y contacto profesional han tenido que ser reformulados y modificados en función de los cambios sociales que se han producido. En este sentido, se ha observado que estamos atendiendo a un número importante de personas en situaciones complejas, tales como:

- Mayores solos o en pareja con carencias de apoyo sociofamiliar o formal de las instituciones públicas.

- Mayores en residencia, las cuales, debido al aislamiento, han creado situaciones de soledad, miedo, incertidumbre, tanto en ellos como en sus familias.

- Familias en situación de necesidad económica.

- Dificultades en las relaciones sociofamiliares como consecuencia del estado de confinamiento, enfermedad o pérdidas personales.

- Secuelas emocionales y físicas, especialmente duelos patológicos.

- Aumento o desarrollo más rápido de enfermedades, debido a la falta de posibilidad de atención profesional (avance de las demencias, perdidas de autonomía 
en las actividades básicas de la vida diaria, salud mental, intervenciones médicas pospuestas).

- Trabajadores que se encontraron en situación de ERTE con pérdida adquisitiva.

- Otras pérdidas de empleo.

- Otras tareas solicitadas por la institución.

Esta situación de restricciones sanitarias ha generado en la ONCE la eliminación de manera temporal de la atención mediante grupos de apoyo presenciales, que suponían una herramienta para promover y favorecer la interacción social y el establecimiento de vínculos de pertenencia y solidaridad entre las personas afiliadas. Herramienta más de intervención y apoyo emocional y social, esencial para la persona. Este tipo de actividad es fundamental, sobre todo cuando se abordan situaciones de soledad y asilamiento, pues el hecho de compartir una historia de vida es un elemento añadido más para poder salir de esas situaciones.

Por el contrario, al replantear la participación de forma telemática, esta ha posibilitado un cierto aumento de participación de personas afiliadas que no podían acudir en otros momentos de forma presencial por falta de acompañamiento o dificultad física. Esto nos hace reflexionar sobre la importancia de combinar los modelos de participación mixtos, adaptados a las necesidades y características de la persona, e implementarlos en el futuro para alcanzar mayor participación e interacción, ampliando así las relaciones, una mayor red y posibilidad de apoyo.

Otra parte importante de nuestro trabajo son las visitas a centros sociosanitarios (residencias, centros de día, hospitales...), que no se han podido realizar, debido a las restricciones que existen para el acceso a este tipo de centros, con lo cual nuestra actuación se ha centrado en el trabajo telefónico. Esta tarea puede realizarse de manera positiva, fundamentalmente por el trabajo previo realizado con estos centros con anterioridad a la pandemia. Los seguimientos realizados, la coordinación y el trabajo en red han sido, y son, fundamentales en este momento en el que es imposible otro tipo de intervención.

Desde nuestra incorporación a la llamada nueva normalidad, no hemos sentido dicha normalidad, pues se detecta un sentimiento de cansancio emocional e incluso 
una pérdida de expectativas de mejora de la situación sanitaria por el alargamiento en el tiempo de la misma. Se mantiene una atención constante ante estos pensamientos que generan desajustes emocionales. Se demuestra que las restricciones en las relaciones sociales, sobre todo en las familiares, hace aumentar la soledad sentida, uno de los mayores retos en este contexto, así como sentimientos de angustia que se regulaban con anterioridad con el apoyo familiar y las rutinas cotidianas.

\section{Aprendizajes}

Al no tener experiencias previas en las que apoyarnos, se generó un aprendizaje a todos los niveles, incrementándose, además, nuestra formación por medio de los webinars organizados por el Consejo y el Colegio de trabajadores sociales, que nos ofrecían herramientas para afrontar esta situación.

La realidad social cambiante, las distintas resoluciones de las administraciones públicas y la gestión emocional se convirtieron en ejes trasversales de nuestro trabajo.

Hemos apoyado a las personas que, por la brecha digital, estaban en desventaja en la tramitación y gestión de recursos, constatando que la precariedad tecnológica y económica, en muchas ocasiones, van de la mano. La imposibilidad de acudir a los distintos organismos para gestionar documentación imprescindible en la tramitación de ayudas, tanto de la ONCE como públicas, ha puesto en evidencia una falta de sensibilidad ante esta vulnerabilidad que hemos tenido que paliar para no aumentar el riesgo de exclusión social.

A pesar de no contar con el contacto físico de la atención presencial, fuimos capaces de establecer lazos emocionales que ayudaron a afrontar situaciones múltiples.

Participamos en un duelo social manifestado por la modificación radical de las normas sociales, expresando la fragilidad del sistema, viviendo el agravamiento de las desigualdades y la aparición de nuevas necesidades, así como la repercusión psicológica por el impacto emocional en la población.

Trabajadoras sociales convertidas, en muchas ocasiones, en el único recurso, pudimos diseñar las acciones necesarias para mejorar las condiciones de los usuarios a los que atendíamos, acompañándoles en su empoderamiento, recono- 
ciendo sus propias herramientas personales para ofrecerles, desde una escucha activa, los mecanismos que paliaran sus sentimientos de aislamiento, soledad, miedo e incertidumbre ante el futuro. Esta atención ha sido considerada positiva por los afiliados, percibiéndonos como un referente para la mejora de su estado anímico.

Los conocimientos teóricos previos que cada profesional tenía en el área de la psicología positiva y en otras áreas han resultado ser bastante útiles, tanto para nosotras mismas, en la gestión de nuestro propio estrés y agotamiento psicológico, como en la atención a nuestros usuarios.

\section{Conclusiones}

Queremos reseñar también que las trabajadoras sociales queremos poner en valor nuestra intervención profesional como imprescindible para proporcionar el soporte emocional, la contención y el acompañamiento a las personas en situaciones de riesgo y vulnerabilidad (situación social dañada, precariedad laboral, pobreza, desigualdad social). Nuestra labor queda, en numerosas ocasiones, invisibilizada o reducida a la gestión de la emergencia o de las prestaciones sociales. Aunque no se vea, compartimos la vida de las personas que atendemos.

Nosotras, como colectivo de trabajo social, atisbamos que el contexto en el que vamos a desarrollar nuestro trabajo se dibuja diferente al contexto previo a la pandemia. Así pues, podemos concluir que nuestra tarea se tendrá que ir adaptando a los cambios, como lo ha hecho hasta ahora. A modo de resumen, ofrecemos nuestros ejes de acción:

1. Con las personas afiliadas:

- Realizar un trabajo de prevención que nos permita anticiparnos a las posibles necesidades que puedan surgir.

- Establecer mejores canales para hacer llegar las demandas de atención, y poder así dar una respuesta más eficaz.

- Agilizar las vías de derivación tanto de los recursos externos como internos.

Felipe, V., Fernández, L., Jiménez, M. C., Martínez, M. J., Mendoza, G., Olalla, J., y Puerto, M.T. (2021). Experiencia de trabajo social en la ONCE en Madrid ante una situación de crisis sanitaria: intervención y aprendizajes. RED Visual: Revista Especializada en Discapacidad Visual, 77, 158-171. https://doi.org/10.53094/DJZE9509. 
- Potenciar conductas de resiliencia, que permitan a la persona enfrentar las dificultades con sus propios recursos, herramientas y habilidades personales.

- Fomentar el autocuidado y los hábitos saludables.

- Potenciar el uso de los medios digitales, así como la formación para poder utilizarlos.

2. Con el equipo de trabajadoras sociales:

- Participar en acciones formativas y de investigación que nos permitan afrontar las nuevas necesidades que surjan.

- Conocer en profundidad las nuevas realidades sociales que han ido surgiendo, en particular todo lo relacionado con el aislamiento social, el miedo al contagio por el virus y las consecuencias del mismo en nuestras vidas.

- Sistematizar el trabajo, unificar criterios y compartir nuevas experiencias.

- Gestionar recursos y prestaciones con fórmulas adaptadas a las circunstancias actuales.

- Mantener el método de acompañamiento social como herramienta clave en este contexto.

- Defender un espacio propio de trabajo, centrado en la intervención social con las personas y su entorno, en la realización de un buen diagnóstico social y una planificación adecuada de la intervención.

- Evaluar el trabajo personal y de equipo, en la línea de mejora continua en la intervención.

- Fomentar la creación de grupos de reflexión, buenas prácticas y supervisión que nos permitan enfrentar las nuevas realidades con nuevos recursos.

- Autocuidado profesional para enfrentarnos adecuadamente al duelo individual y colectivo. 
Para finalizar, compartimos una reflexión sobre la adaptación del colectivo del trabajo social a los cambios sociales, adaptándose a todas las situaciones de conflicto, confusión, miedo o falta de expectativas, reinventado su labor en el día a día y defendiendo los derechos de las personas.

Esta situación de crisis sanitaria está llevando a nuestra sociedad a un cansancio que nos hace necesario seguir reformulando nuestra actuación profesional, con un alto nivel de motivación, la necesidad de un cuidado profesional permanente y una gran agilidad para enfrentarnos a la nueva situación de crisis, en la que es fácil confundir la emergencia con la urgencia. Cualquier actuación por nuestra parte puede modificar y/o condicionar un aspecto vital de la vida de estas personas, motivo por el cual debemos mantenernos alerta, en constante formación y actualizados con todas aquellas nuevas situaciones que, como profesionales, se nos presenten.

Tuvimos una sensación de soledad profesional, paliada con el sentimiento de apoyo entre las compañeras.

Empoderar a las personas nos dará la satisfacción de un trabajo bien hecho donde dejaremos de ser «padres» para ser «maestros».

Cuando el «agotamiento pandémico» está ya en boca de todos, terminamos con una cita de Seligman (Alconada, 2021), padre de la psicología positiva, en la que decía que «El mundo está de parto y la pregunta ahora es qué va a dar a luz».

\section{Referencias bibliográficas}

Aguilar, M. J. (2013). Trabajo social: concepto y metodología. Paraninfo.

Alconada, H. (23 de febrero de 2021). El mundo está de parto ahora y la pregunta es qué va a dar a luz. El Tiempo. https://www.eltiempo.com/salud/entrevista-a-martin-seligmanel-mundo-esta-de-parto-ahora-y-la-pregunta-es-que-va-a-dar-a-luz-568804.

Consejo General del Trabajo Social (2015). Código deontológico de trabajo social. Segunda edición actualizada. https://www.cgtrabajosocial.es/codigo_deontologico. 
Organización Nacional de Ciegos Españoles (1998). Nuevo modelo de prestación de Servicios Sociales en la ONCE. Documento de uso interno.

Pérez, J.D. (2019). Efecto en el desarrollo y/o bienestar social en América Latina como consecuencia de la expansión de la corrupción [PDF]. Trabajo de investigación para la obtención del grado. Universidad San Ignacio de Loyola. http://repositorio.usil.edu.pe/ handle/USIL/9825.

Zamanillo, T. (1989). Bienestar social y trabajo social: tendencias actuales [PDF]. Cuadernos de Trabajo Social, 2, 9-21. https://revistas.ucm.es/index.php/CUTS/issue/view/CUTS898911.

Vanesa Felipe López. Trabajadora social. Delegación Territorial de la ONCE en Madrid. Prim, 3; 28004 Madrid (España). Correo electrónico: vfl@once.es.

Laura Fernández Sanz. Trabajadora social. Delegación Territorial de la ONCE en Madrid. Agencia de Vallecas. Picos de Europa, 46; 28038 Madrid (España).Correo electrónico: lfs@once.es.

M. Carmen Jiménez Cruz. Trabajadora social en la Agencia de Vallecas y trabajadora social asesora en la Dirección General de la ONCE. Calle del Prado, 24; 28014 Madrid (España). Correo electrónico:mcajc@once.es.

M. ${ }^{a}$ José Martínez Vitoria. Trabajadora social. Delegación Territorial de la ONCE en Madrid. Prim, 3; 28004 Madrid (España). Correo electrónico: mmvi@once.es.

Guacimara Mendoza Mendoza. Trabajadora social. Delegación Territorial de la ONCE en Madrid. Agencia de Ciudad Lineal. Doctor Vallejo, 4-6; 28027 Madrid (España). Correo electrónico: gumm@once.es.

Julia Olalla Suárez. Trabajadora social. Delegación Territorial de la ONCE en Madrid. Prim, 3; 28004 Madrid (España).Correo electrónico: jos@once.es.

M. ${ }^{a}$ Teresa Puerto Simón. Trabajadora social. Delegación Territorial de la ONCE en Madrid. Agencia de Getafe, Aranjuez y Leganés. Álvaro de Bazán, 14; 28902 Getafe (Madrid, España). Correo electrónico: tps@once.es. 